PEIXOTO, J.R.; MATHIAS FILHO, L.; SILVA, C.M.; OLIVEIRA, C.M.; CECILIO FILHO, A.B. Produção de genótipos de tomateiro tipo 'Salada' no período de inverno, em Araguari. Horticultura Brasileira, Brasília, v. 19, n. 2, p. 148-150, julho 2.001.

\title{
Produção de genótipos de tomateiro tipo 'Salada' no período de inverno, em Araguari.
}

\author{
José Ricardo Peixoto ${ }^{1}$; Lourival Mathias Filho²; Célio M. Silva²; Carlos M. Oliveira ${ }^{3}$; Arthur Bernardes \\ Cecilio Filho ${ }^{4}$ \\ ${ }^{1}$ UnB, FAV, C. Postal 04508, 70.910-900 Brasília-DF, e-mail: peixoto@ unb.br, ${ }^{2}$ UFU, C. Postal 593, 38400-902 Uberlândia-MG. ${ }^{3}$ UFLA, \\ C. Postal 37, 37.200-902 Lavras-MG. ${ }^{4}$ UNESP, FCAV, C. Postal 04500, 14870-000 Jaboticabal, SP.
}

\section{RESUMO}

O trabalho foi desenvolvido em Araguari (MG), na época de inverno, com o objetivo de verificar o desempenho agronômico de genótipos de tomateiro tipo 'Salada'. Utilizou-se o delineamento experimental de blocos casualizados com 18 tratamentos e quatro repetições. As colheitas iniciaram 76 dias após o transplante, de 03/ 08/1996 a 05/10/1996, para a maioria dos genótipos, sendo feito um total de 18 colheitas. Como resultado os genótipos T-8, T-10, Barão Vermelho AG-561, Carmen, Agora e Olimpo superaram significativamente as demais em produtividade comercial, variando de 125,3 t/ha (Olimpo) a 142,6 t/ha (T-8), sendo portanto recomendáveis para a região, no período de inverno. Mais de $60 \%$ dos genótipos tiveram frutos com peso médio superior a $200 \mathrm{~g}$, com destaque para Barão Vermelho AG-561 (259,50 g) e Sunbolt (255,75 g) que apresentaram mais de $30 \%$ de frutos do tipo extra AA, sendo superados apenas pelo genótipo Carmen, porém com padrão de fruto bem diferente em tamanho. Com mais de $44 \%$ de frutos tipo extra A destacaram-se os genótipos Super Marmande, T-8, Sunbeam e AG-233. O genótipo Agora destacou-se com frutos do tipo primeira $(59,70 \%)$.

Palavras-chave: Lycopersicon esculentum, rendimento, classificação.

\begin{abstract}
Agronomic characteristics of tomato genotypes ('Salad' type) during winter season, in Araguari, Minas Gerais.

A field trial was carried out in Araguari, Brazil, during the winter season, to evaluate the agronomic potential of eighteen tomato genotypes ('Salad' type). A randomized blocks design with four replications was used. A total of 18 harvests were carried out, beginning 76 days after transplanting, from August, $3^{\text {rd }}$ to October, $5^{\text {th }}, 1996$. In general, the genotypes T-8, T-10, Barão Vermelho AG561, Carmen, Agora and Olimpo presented higher yields, varying from $125.3 \mathrm{t} / \mathrm{ha}$ (Olimpo) to $142.6 \mathrm{t} / \mathrm{ha}(\mathrm{T}-8)$. These genotypes can be cultivated in this region, during the winter season. More than $60 \%$ of the tomato genotypes presented fruits with $200 \mathrm{~g}$, the outstanding one being the Barão Vermelho AG-561 (259,50 g) and Sunbolt $(255,75 \mathrm{~g})$, with more than $30 \%$ of type extra AA. 'Carmen' was superior, indeed the fruits were very irregular in size. Genotypes Super Marmande, T-8, Sunbeam and AG-233 presented more than $44 \%$ of extra A fruits. Genotype Agora $(59,70 \%)$ was outstanding, equal to the first type.
\end{abstract}

Keywords: Lycopersicon esculentum, yield, classification.

\section{(Aceito para publicação em 17 de maio de 2.001)}

A cadeia produtiva do tomate passa atualmente por importantes mudanças, orientadas para sua modernização. Os principais ajustes se referem à segmentação varietal, à incorporação de novas tecnologias de produção, à mudanças nos canais de comercialização e setor de embalagens e à expansão das redes de fast food (Melo, 1997).

No que diz respeito ao panorama de cultivares, a Santa Clara ainda é líder absoluta com mais de $70 \%$ do mercado, com frutos do tipo 'Santa Cruz'. No entanto, a tendência é que essa cultivar ceda cada vez mais espaço a outros tipos de genótipos, de introdução recente. Merece destaque o crescimento da nova geração de híbridos do tipo 'Salada ou Caqui', que substituíram as tradicionais cultivares japonesas de frutos exageradamente grandes e moles. Os tomates do tipo longa-vida e os extrafirmes também têm mostrado ex- pansão em ritmo mais acelerado do que se previa, sobretudo nas zonas de produção do Sudeste e do Sul (Melo, 1997). Todavia há preferência do consumidor para cultivares de melhor qualidade.

Atualmente a ênfase maior no melhoramento visa a obtenção de híbridos $F_{1}$, pela série de vantagens que apresentam, como produtividade e qualidade, precocidade, resistência a doenças e pragas, uniformidade, entre outras características. O uso de cultivares, híbridos $F_{1}$ ou não, bastante produtivos e geneticamente resistentes a patógenos (inclusive a nematóides) e a pragas, constitui a alternativa ideal, segundo pesquisadores, técnicos e agricultores. Tais cultivares apresentam a solução, muitas vezes duradoura, para certos problemas fitossanitários, acessíveis à maioria dos agricultores e permitem reduzir a poluição do ambiente (Ferraz \& Mendes, 1992).
Pouca ênfase tem sido dada aos estudos de avaliação agronômica de genótipos, nas mesmas condições edafoclimáticas, visando recomendar os melhores para determinada região e sistema de cultivo. A avaliação de características agronômicas, tem oferecido importantes contribuições no que diz respeito à adaptação edafoclimática de genótipos com potencial nas diversas regiões produtoras do Brasil (Leal, 1973; Faria, 1997).

O objetivo deste trabalho foi avaliar agronomicamente 18 genótipos de tomate tipo 'Salada', no período de inverno, em Araguari-MG.

\section{MATERIAL E MÉTODOS}

O experimento foi instalado e conduzido na Fazenda Jordão, localizada no município de Araguari, no período de inverno. Este município está localizado 
Tabela 1. Características agronômicas de 18 genótipos de tomate tipo 'Salada' cultivados no período de inverno em Araguari. Uberlândia, UFU, $1996^{1}$.

\begin{tabular}{lrlc}
\hline Genótipos & $\begin{array}{c}\text { Rendimento } \\
\text { comercial } \\
\text { (t/ha) }\end{array}$ & $\begin{array}{c}\text { Peso médio } \\
\text { dos frutos }(\mathbf{g})\end{array}$ & $\begin{array}{c}\text { No de } \\
\text { frutos/planta }\end{array}$ \\
\hline T-8 & $142,60 \mathrm{a}$ & $253,00 \mathrm{abc}$ & $33,82 \mathrm{de}$ \\
T-10 & $140,15 \mathrm{a}$ & $234,75 \mathrm{abcd}$ & $35,65 \mathrm{~cd}$ \\
Barão Vermelho AG-561 & $132,20 \mathrm{a}$ & $259,50 \mathrm{a}$ & $30,78 \mathrm{def}$ \\
Carmen & $132,07 \mathrm{a}$ & $127,25 \mathrm{~g}$ & $62,18 \mathrm{a}$ \\
Agora & $126,40 \mathrm{a}$ & $180,75 \mathrm{ef}$ & $41,85 \mathrm{bc}$ \\
Olimpo & $125,31 \mathrm{a}$ & $230,50 \mathrm{abcd}$ & $32,60 \mathrm{def}$ \\
AG -233 & $95,83 \mathrm{~b}$ & $129,25 \mathrm{~g}$ & $44,45 \mathrm{~b}$ \\
Sunbeam & $93,39 \mathrm{~b}$ & $224,75 \mathrm{abcde}$ & $24,98 \mathrm{fgh}$ \\
Cinthia & $92,68 \mathrm{~b}$ & $149,50 \mathrm{fg}$ & $37,22 \mathrm{bcd}$ \\
Empire & $91,59 \mathrm{~b}$ & $253,50 \mathrm{ab}$ & $21,75 \mathrm{gh}$ \\
Sunjay & $88,25 \mathrm{~b}$ & $216,75 \mathrm{abcde}$ & $25,13 \mathrm{fgh}$ \\
Super Marmande & $88,15 \mathrm{~b}$ & $142,00 \mathrm{fg}$ & $37,22 \mathrm{bcd}$ \\
Pacific & $86,33 \mathrm{~b}$ & $207,75 \mathrm{bcde}$ & $25,08 \mathrm{fgh}$ \\
Sunny & $83,00 \mathrm{~b}$ & $198,25 \mathrm{de}$ & $25,15 \mathrm{fgh}$ \\
EF-52 & $80,81 \mathrm{~b}$ & $179,00 \mathrm{ef}$ & $27,28 \mathrm{efg}$ \\
EF-49 & $78,68 \mathrm{~b}$ & $237,00 \mathrm{abcd}$ & $21,35 \mathrm{gh}$ \\
Sunbolt & $77,71 \mathrm{~b}$ & $255,75 \mathrm{a}$ & $18,25 \mathrm{~h}$ \\
EF-50 & $73,91 \mathrm{~b}$ & $205,50 \mathrm{cde}$ & $19,88 \mathrm{gh}$ \\
\hline CV (\%) & 8,73 & 8,95 & 9,35 \\
\hline
\end{tabular}

${ }^{1}$ Médias seguidas pela mesma letra, nas colunas, não diferem estatisticamente, entre si, pelo teste de Tukey, a $5 \%$ de probabilidade.

entre $505 \mathrm{~m}$ e $1087 \mathrm{~m}$ de altitude com temperatura média anual de $20,7^{\circ} \mathrm{C}$, sendo a média mínima anual de $16^{\circ} \mathrm{C}$, e a média máxima anual de $26,3^{\circ} \mathrm{C}$. A precipitação média anual é de $1641 \mathrm{~mm}$.

Foram utilizados os genótipos Barão Vermelho AG-561 (PA) e Super Marmande (PA) com hábito de crescimento indeterminado e mais 16 híbridos $\mathrm{F}_{1}$, sendo Agora, Carmen, Cinthia, Olimpo, T-8 e T-10 com crescimento indeterminado e EF-49, EF-50, EF-52, Empire, AG-233, Pacific, Sunbeam, Sunbolt, Sunjay e Sunny com crescimento determinado.

A adubação de plantio foi feita de acordo com a análise química do solo, que apresentou: $\mathrm{pH}$ (água) $=6,0 ; \mathrm{P}=$ $4,0 \mathrm{mg} / \mathrm{dm}^{3} ; \mathrm{K}=160 \mathrm{mg} / \mathrm{dm}^{3} ; \mathrm{Al}=0,0$ $\mathrm{cmol} / \mathrm{dm}^{3} ; \mathrm{Ca}=4,6 \mathrm{cmol} / \mathrm{dm}^{3} ; \mathrm{Mg}=1,4$ $\mathrm{cmol} / \mathrm{dm}^{3} ; \mathrm{H}+\mathrm{Al}=2,6 \mathrm{cmol} / \mathrm{dm}^{3} ; \mathrm{SB}$ (Soma de Bases) $=6,4 \mathrm{cmol} / \mathrm{dm}^{3} ; \mathrm{t}(\mathrm{CTC}$ efetiva/CTC a pH 7,0) $=6,0 \mathrm{cmol} / \mathrm{dm}^{3}$; $\mathrm{T}=9,0 \mathrm{cmol} / \mathrm{dm}^{3} ; \mathrm{V}=71 \% ; \mathrm{m}$ (Sat. de $\mathrm{Al})=0 \%$ e matéria orgânica - M.O.
$($ Walkley-Black $)=2,9 \mathrm{dag} / \mathrm{kg}$. No plantio, em cada metro linear de sulco, foram utilizados aproximadamente $2 \mathrm{~kg}$ de esterco de curral curtido, $300 \mathrm{~g}$ de superfosfato simples, $30 \mathrm{~g}$ de cloreto de potássio e $10 \mathrm{~g}$ de FTE. Realizaram-se quatro adubações de cobertura, via solo, a partir dos 15 dias do transplante e repetindo a cada 14 dias, utilizando-se 25 g/planta de sulfato de amônio, $25 \mathrm{~g} / \mathrm{plan}$ ta de nitrocálcio e $15 \mathrm{~g} /$ planta de cloreto de potássio, e foliar (Orgamin a 0,02\% e Ouro Verde a $0,01 \%$ ) em cada aplicação. As mudas foram formadas em bandejas de poliestireno com 128 células. O substrato utilizado foi composto por vermiculita e casca de Pinus. O transplante foi feito para o campo, recém preparado ( 2 arações e uma gradagem) e calcariado, utilizando-se o espaçamento $1,0 \times 0,6 \mathrm{~m}$, com 2 plantas/cova, conduzidas deixando uma haste por planta, no sistema de cerca cruzada, para todos os genótipos testados. Efetuaramse duas operações de desbaste, deixando em média 4 frutos/penca.

Realizaram-se pulverizações preventivas e curativas semanais, com fungicidas à base de cobre, mancozeb, metalaxyl e tebuconazole e inseticidas fisiológicos, fosforados e piretróides, visando o controle de patógenos e pragas. Foram efetuados todos os tratos culturais indispensáveis à cultura. A irrigação foi feita pelo método de infiltração, utilizando turno de rega de 2 dias, sem controle da lâmina de água. O delineamento experimental utilizado foi de blocos casualizados, com quatro repetições e 18 tratamentos, sendo cada parcela útil constituída de 24 plantas.

As colheitas, efetuadas duas vezes por semana somaram um total de 18 colheitas, de 76 a 136 dias do transplantio. Avaliaram-se a produtividade comercial, peso médio e número de frutos por planta. Os frutos foram classificados em extra AA, extra A e primeira, de acordo com o mercado local, baseado no aspecto e tamanho dos frutos.

Todos os dados originais foram submetidos à análise de variância (Pimentel-Gomes, 1978), comparandose as médias pelo teste de Tukey, a 5\% de probabilidade.

\section{RESULTADOS E DISCUSSÃO}

Os genótipos T-8, T-10, Barão Vermelho AG-561, Carmen, Agora e Olimpo superaram significativamente os demais, em rendimento comercial, variando de 125,3 t/ha (Olimpo) a 142,6 t/ha (T-8). Para número total de frutos/planta destacaram-se Carmen, AG-233, Agora, Cinthia e Super Marmande (Tabela 1).

Mais de 60\% dos genótipos tiveram peso médio de frutos superior a $200 \mathrm{~g}$, com destaque para Barão Vermelho AG561, T-8, Sunbolt e Empire com mais de $250 \mathrm{~g}$ (Tabela 1). Carmen (padrão de fruto bem diferente em tamanho) apresentou a maior percentagem de frutos tipo extra AA $(41,2 \%)$, seguido por Barão Vermelho AG-561 e Sunbolt com $31,6 \%$ e $30,4 \%$, respectivamente, porém diferente do Carmen. Com mais de $44 \%$ de frutos tipo extra A destacaram-se os genótipos Super Marmande, T-8, Sunbeam e AG-233. Porém, diferiram somente de Carmen e Agora. Além dos genótipos Agora, com 59,7\% de frutos do tipo primeira, destacaram-se ainda 
Cinthia, AG-233, AF-52, Sunny e Pacific (Tabela 2).

Faria (1997) avaliando 16 genótipos no período de verão, também em Araguari-MG, sendo 9 genótipos iguais aos deste trabalho (T-8, EF-52, Agora, Empire, Carmen, Pacific, Sunny, Sunbolt e Cinthia) obtiveram rendimento e pesos médios inferiores, inclusive no genótipo T-8 com rendimento total de 95,69 t/ha, rendimento comercial de 85,69 t/ha e peso médio de 220,5 gramas. Houve grandes diferenças também nas percentagens de frutos dos tipos extra AA, extra A e primeira. Este menor desempenho dos genótipos de tomate no período de verão, se deve possivelmente, à maior incidência e severidade de patógenos neste período, prejudicando o rendimento e qualidade dos mesmos.

Oliveira \& Araújo (1998) avaliaram quatro híbridos (EF-49, EF-50, EF-52 e Saladinha) e duas cultivares (Santa Adélia Super e IPA-5) nas condições de verão, em Areia $(\mathrm{PB})$ e verificaram que os híbridos EF-5- e EF-52 apresentaram maior produção total e peso médio de frutos, no verão daquela região.

Os genótipos Cinthia e Agora, apesar de terem frutos de menor tamanho, foram comparados aos tomates considerados 'graúdos', em função do mercado ter pouco conhecimento de suas características, e dessa forma, não são classificados como padrão 'Carmen' (longa vida). Porém, se comparássemos com este padrão, certamente teríamos uma alta percentagem de frutos tipo extra AA.

Vários genótipos apresentaram características agronômicas bastante favoráveis associadas ao peso médio, destacando-se T-8, T-10, Barão Vermelho AG-561, Carmen, Olimpo, Empire, Sunbeam e Sunjay, podendo ser recomendados para o plantio de inverno na região de Araguari (MG), caso se confirme a superioridade dos mesmos num segundo ensaio, nas mesmas condições climáticas e edáficas. Ressalta-se que, apesar de Cinthia ter apresentado um rendimento apenas razoável, comparativamente ao melhores genótipos, este apresentou aparentemente uma excelente

Tabela 2. Classificação comercial de 18 genótipos de tomate tipo 'Salada' cultivados no período de inverno em Araguari. Uberlândia, UFU, $1996^{1}$.

\begin{tabular}{llll}
\hline \multicolumn{1}{c}{ Genótipos } & $\begin{array}{l}\text { Frutos tipo } \\
\text { extra AA (\%) }\end{array}$ & $\begin{array}{c}\text { Frutos tipo } \\
\text { extra A (\%) }\end{array}$ & $\begin{array}{c}\text { Frutos tipo } \\
\text { primeira (\%) }\end{array}$ \\
\hline T-8 & $26,33 \mathrm{bc}$ & $44,72 \mathrm{a}$ & $28,95 \mathrm{fg}$ \\
T-10 & $23,93 \mathrm{bcd}$ & $41,72 \mathrm{ab}$ & $34,35 \mathrm{efg}$ \\
Barão Vermelho AG-561 & $31,63 \mathrm{~b}$ & $38,88 \mathrm{ab}$ & $29,50 \mathrm{fg}$ \\
Carmen & $41,15 \mathrm{a}$ & $33,22 \mathrm{~b}$ & $25,63 \mathrm{~g}$ \\
Agora & $7,78 \mathrm{hij}$ & $32,53 \mathrm{~b}$ & $59,70 \mathrm{a}$ \\
Olimpo & $16,43 \mathrm{defgh}$ & $42,53 \mathrm{ab}$ & $41,05 \mathrm{cdefg}$ \\
AG-233 & $0,53 \mathrm{j}$ & $44,53 \mathrm{a}$ & $54,95 \mathrm{abc}$ \\
Sunbeam & $22,78 \mathrm{bcde}$ & $44,67 \mathrm{a}$ & $32,55 \mathrm{efg}$ \\
Cinthia & $1,95 \mathrm{ij}$ & $40,68 \mathrm{ab}$ & $57,38 \mathrm{ab}$ \\
Empire & $29,23 \mathrm{~b}$ & $42,58 \mathrm{ab}$ & $28,20 \mathrm{fg}$ \\
Sunjay & $16,38 \mathrm{defgh}$ & $42,55 \mathrm{ab}$ & $41,08 \mathrm{cdefg}$ \\
Super Marmande & $23,40 \mathrm{bcd}$ & $47,05 \mathrm{a}$ & $29,55 \mathrm{fg}$ \\
Pacific & $13,88 \mathrm{efgh}$ & $39,53 \mathrm{ab}$ & $46,60 \mathrm{abcde}$ \\
Sunny & $10,28 \mathrm{fghi}$ & $42,30 \mathrm{ab}$ & $47,42 \mathrm{abcde}$ \\
EF-52 & $8,80 \mathrm{ghij}$ & $38,25 \mathrm{ab}$ & $52,95 \mathrm{abcd}$ \\
EF-49 & $17,75 \mathrm{cdefg}$ & $39,92 \mathrm{ab}$ & $42,45 \mathrm{bcdef}$ \\
Sunbolt & $30,38 \mathrm{~b}$ & $39,75 \mathrm{ab}$ & $29,88 \mathrm{fg}$ \\
EF-50 & $18,95 \mathrm{cdef}$ & $42,30 \mathrm{ab}$ & $38,75 \mathrm{defg}$ \\
\hline CV (\%) & 18,16 & 10,51 & 14,88 \\
\hline
\end{tabular}

${ }^{1}$ Médias seguidas pela mesma letra, nas colunas, não diferem estatisticamente, entre si, pelo teste de Tukey, a 5\% de probabilidade.

firmeza e poucos sintomas visuais de incidência de patógenos, durante todo o período de condução do experimento, ao contrário dos demais genótipos. Ressalta-se também a potencialidade demonstrada pelo genótipo Agora, que juntamente com Cinthia, apresentaram formato e peso médio do fruto comparável ao padrão 'Carmen'.

\section{AGRADECIMENTOS}

Os autores agradecem às empresas que forneceram as sementes (HortiAgro, Agroceres, Agroflora, Topseed, Isla e Asgrow) e aos Senhores: Rui Alves Peixoto (Produtor Rural), Rui Alves Peixoto Júnior, Joaquim Lopes (Auxiliar Técnico da UFU), Rinaldo Alves Peixoto, Vanderlei Batista da Silva e Júnio Batista Carneiro pela valiosa ajuda na instalação, condução e avaliações do experimento.

\section{LITERATURA CITADA}

FARIA, V.R.C. Avaliação de genótipos de tomate tipo "Salada", no período de verão em Araguari, MG. Uberlândia: UFU, 1997. 34 p. (Monografia graduação).

FERRAZ, F.; MENDES, M.L. O nematóide das galhas. In: Informe Agropecuário - nematóides: o inimigo oculto da agricultura. Belo Horizonte, v. 16, n. 172 , p. 43-45, 1992.

LEAL, N.R. Comparação da produtividade da cultivar de tomate "Alcobaça" com três cultivares do tipo "Santa cruz", na Baixada Fluminense. Revista Ceres, Viçosa, v. 20, n. 107, p. 65-67, jan./ mar. 1973.

MELO, P.C.T. Do canteiro à mesa, muitas novidades. In: Agrianual 97 - Anuário da Agricultura Brasileira, FNP Consultoria e Comércio, 1997. p. 402-404.

OLIVEIRA, A.P.; ARAUJO, J.C. Desempenho de tomates híbridos nas condições de verão, em Areia-PB. Honticultura Brasileira, Brasília, v. 16, n. 2, p. 176-177, 1998.

PIMENTEL-GOMES, F. Curso de Estatística Experimental. 8. ed. São Paulo: Nobel, 1978. 430 p. 